\title{
Hard to Boil Massecuite
}

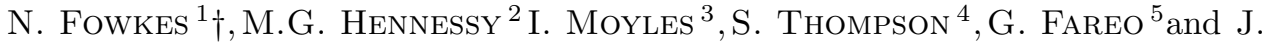 \\ ATHERFOLD $^{5}$ \\ ${ }^{1}$ University of Western Australia \\ ${ }^{2}$ CRM, Barcelona \\ ${ }^{3}$ University of Limerick \\ ${ }^{4}$ University of Oxford \\ ${ }^{5}$ University of the Witwatersrand
}

(Communicated to MIIR on 12 April 2021)

Study Group: MISGSA2017. 16-20 January 2017, AIMS, Cape Town.

Communicated by: Graeme Hocking

Industrial Partner: Sugar Milling Research Institute

Presenter: R. C. Loubser

Team Members: J. Atherfold, Witwatersrand; Z. Dlamini, Pretoria; G. Fareo, Witswatersrand; N. Fowkes, Perth; M.G. Hennessy, Barcelona; Meghan Kennealy, Stellenbosch; I. Moyles, Limerick; Colin Please, Oxford; Tim Myers, Barcelona; T. Motsepa, Mmabatho; S.O. Ojako, KwaZulu-Natal; A. Nour; V. Magagula, Eswatini.

Industrial Sector: Food and Drink

Tools: Heat transfer, two-phase flow, nucleation

Key Words: Sugar, Boiling, Massecuite, Bubbles

MSC2020 Codes: 74, 34, 35

\section{Summary}

Evaporators are used to remove most of the water from sugar cane juice leaving a syrup which is fed into the pan boiler. Seed crystals are then added to the syrup and the mixture (referred to as massecuite) is further heated to remove moisture so that sugar crystals grow. The pan boiling process normally works smoothly. However in the South African harvesting season (the wet season) the syrup does not appear to boil and crystallisation is significantly slowed. The problem is thought to be due to the presence of impurities (specifically polysaccharides) which change the rheological properties of the massecuite. A range of models were produced to examine the effects of changes in massecuite properties on the operation on the boiler and the boiling process itself. The results suggest that the primary cause of hard to boil massecuite is bubble suppression although other mechanisms may also be involved.

$\dagger$ Corresponding Author: neville.fowkes@uwa.edu.au 


\section{Introduction}

\subsection{The sugar production process}

Cane stalks are shredded and squeezed to extract their natural juice, and additional juice is flushed out by draining water through the crushed fibres in a diffuser. Evaporators are then used to remove excess water leaving a syrup consisting of $60 \%$ dry solids and $40 \%$ water. The syrup is then fed into a crystallisation pan where seed crystals are added and further evaporation occurs in a process referred to as pan boiling. In the pan boiler the two phase mixture, now referred to as massecuite, is maintained in a meta-stable state of supersaturation such that the sucrose deposits onto existing crystal surfaces; homogeneous nucleation does not occur. The sugar crystals grow and begin to settle and then the mixture is removed from the boiler. The crystals in molasses mixture is then sent to a rapidly spinning centrifuge to remove the molasses, leaving pure naturally white sugar crystals. The sugar crystals are then dried. The whole process is carefully controlled to ensure that crystals are pure and of the required size.

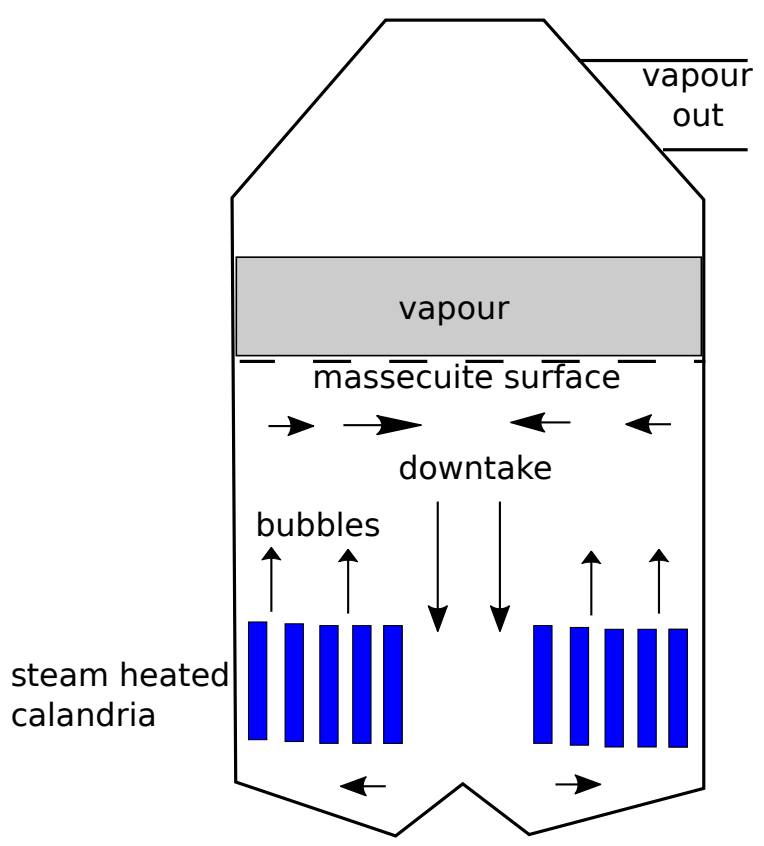

Figure 1: A pan boiler. The heated calandria drives a convective flow in the massecuite. Bubbles formed within the heated calandria tubes escape through the massecuite surface and the vapour is removed from the boiler. 

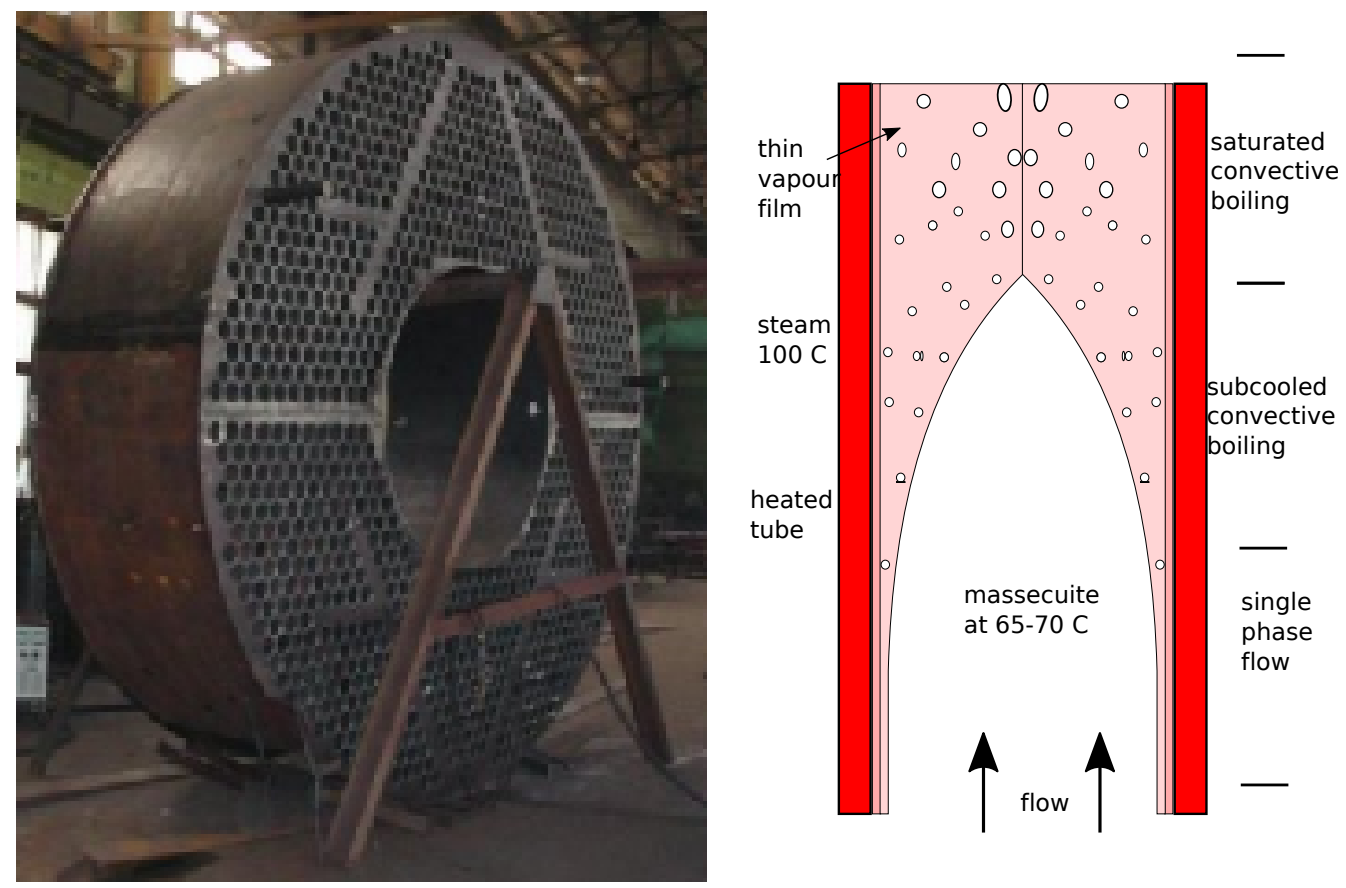

Figure 2: Left: A calandria heat exchanger. Right: Massecuite boiling in the lower end of a calandria tube.

Our interest is in the pan boiler. Pan boiler designs vary enormously but a typical structure will be described, see Figure 1. The boiler is a fully enclosed container of volume $45 \mathrm{~m}^{3}$, sectional area of $223 \mathrm{~m}^{2}$, and height $5-6 \mathrm{~m}$. Within the boiler is a calandria. The calandria is a heat exchanger which consists of 1,200 vertical open metal tubes (typical diameter $100 \mathrm{~mm}$ and length $1 \mathrm{~m}$ ) encased in a fully enclosed metal shell with an external cylindrical tube shape that fills the boiler, see Figure 2 Left. Heated steam (temperature $110^{\circ} \mathrm{C}$ ) is circulated through the calandria shell and thus around the outside of the cylindrical tubes so that the tubes are maintained at the steam temperature. The calandria is fully submerged in the massecuite pool within the boiler so that massecuite fills the tubes and is heated by conduction through the tube walls. The massecuite in the tubes boils and the bubbles rise up and exit the tubes, and then rise to the surface of the massecuite pool where they burst with the water vapour extracted from the top of the boiler, see Figure 1. The bubbles carry massecuite up and out the tubes and so the pool of massecuite is cycled through the calandria. A natural convection pattern is set up in the boiler with pooled massecuite being drawn up into the calandria tubes and then sinking down the axis of the 
calandria. This recirculation process enables all the massecuite to cycle through the tubes several times before completion and is assisted by mechanical stirring in the form of a scraper below the calandria. The whole boiler is maintained at below atmospheric pressure so that the massecuite boils at a temperature in the range $70-80^{\circ} \mathrm{C}$; somewhat lower than $100^{\circ} \mathrm{C}$. Seed crystals and water are fed into the boiler in a carefully controlled way to ensure the steady growth of sugar crystals until the required size is reached and then the massecuite is dumped out and sent to the centrifuge. The processing time under normal conditions is about 20 mins. For a more detailed description of the process see Ziegler [1].

\subsection{Boiling problems}

Under normal circumstances the pan boiling process works smoothly, but this does require careful management of the temperatures and pressures within the boiler and the feed of seed crystals. The harvesting season in South Africa lasts from March to December and there are no problems until about November. After that boiling difficulties can arise. Specifically vapour bubbles (appear) to not form and crystal growth is significantly slowed, and at times halted completely. Also an increase in massecuite viscosity is observed which is thought to result in poor circulation into and out of the calandria tubes.

It should be noted that during the early and mid harvest period the cane is quickly transported and processed. In the late season (November-December), however, rain falls and harvesting equipment cannot operate in the rain so that there are delays between the burning (to remove loose leaves), harvesting, transport and crushing of the cane. It is the impurities that arise in the cane due to this delay that are thought to be responsible for hard-to-boil (HTB) massecuite; to date no specific cause has been identified or reliable cure found. It is known that after burning the cane deteriorates due to microbial action and in particular polysaccharides are produced and these are known to effect the rheological properties of the massecuite. The objective of the MISG was to understand the effect of changes in the massecuite properties on the boiling and crystallisation process and if possible to identify the key parameters. One might hope that such an identification would enable useful experiments to be designed, but also cures for unsticking the process might be suggested.

In Section 2 simple models of the heat exchanges and fluid dynamical processes occurring in the calandria are examined and a few key parameters identified. This work, as well as work from previous studies, suggests that it is the difficulty of bubble formation and escape from the changed massecuite that causes the problem; a series of models are developed for examining these issues in Section 3. Concluding remarks and made in Section 4. Before doing all this we will briefly survey relevant work on the hard to boil (HTB) massecuite problem. 


\subsection{Previous work on boiling difficulties}

It is not surprising that there has been a great deal of experimentation and speculation concerning this expensive problem which occurs world wide and many suggestions have been made to explain and overcome the 'stuck process' when it happens. The problem can be mild to severe, see Koster et al [2] and can be so severe that it can cause a factory stand-still, which occurred at New Iberia, LA factory in 2002 for three weeks.

Saska [3] (2003) measured the heat transfer coefficients of syrups, molasses and massecuites boiled in pilot vacuum pans and found that some Louisanna mill samples had less than a one tenth of the heat transfer capability of normal samples. They reported that the reduced heat transfer rates were unrelated to the crystallization kinetics and the presence of the common polysaccharides dextran and starch. Surfactants, lubricants, and soda ash also appeared to have little or no effect on improving heat transfer in the HTB samples and the viscosity of the samples appeared to be unaffected. Eggleston, Côte and Santee [5] reported a 9-33\% lower heat conductivity of HTB massecuite in Louisianna mills in the USA. Also they reported that a highly viscous intermolecular network was present in HTB molasses which would explain the difficulty in removing entrapped water on boiling. A variety of chemical agents have been tested (soda ash, sodium hydrosulfite, melassigenic compounds) to improve the boiling characteristics of massecuite (viscosity reduction, sucrose solubility agents, surfactants,...) with very limited success [9]; when a HTB problem occurs there appears to be little a mill can do to correct it.

The above results are somewhat confusing and even perhaps contradictory, which may be because the experiments were carried out in circumstances well removed to those occurring within the boiler; the flow and mixing regime is very different in a calandria tube than in an open container. However Rouillard [4] and Rein [6] have carried out experiments using a vertical plastic tube arrangement similar to that occurring within the boiler. The tube was filled with corn syrup, and air was injected at the bottom of the tube caused the syrup to move up and out of the tube, to be returned to the bottom of the tube in a return pipe. The viscosity of the syrup was varied. The various two phase flow regimes described below were observed. Fluctuations in pressure in the enclosed vessel occurred over periods of the order of 3 or 4 secs which is consistent with slug flow behaviour. Follow-up and much more detailed lab-scale experiments were carried out by Echeverri [11] who again bubbled air through both water and high viscosity corn syrup in tubes. He also used an experimental lab rig to examine the overall convection pattern developed in the pan boiler and used the results to calibrate a CFD model. All the above experimental results were performed using liquids whose viscosity was significantly less (0.2 to 3.9 Pa.sec) than that of massecuite (1-14 Pa.sec) in the pan boiler, however the results obtained are consistent with results for two phase flow obtained in many other contexts. 


\subsection{Two phase flow models}

Much experimental and theoretical work has been done on two phase flow models, both with and without phase change as in boiling, and understandably water and air are the fluids of choice. The classical engineering texts on such flows are Butterworth and Hewitt [7] and Wallis [8]. Of particular relevance in the present context are the water/air 'bubble' flows in a vertical tube. Various flow regimes are observed depending on the volume fluxes of air and water, see Butterworth and Hewitt [7]. With increasing air flux levels (in the absence of boiling) one observes:

1. Bubble flow with a dispersion of bubbles in a water continuum.

2. Slug or plug flow where bubbles coalescence occurs and the bubbles essentially fill the pipe and alternating slugs of liquid and air pass down the pipe.

3. Churn flow where the plugs break down and gas tubes appear.

4. Annular flow whereby liquid flows along the wall in a thin film with gas phase flow in the centre.

If boiling occurs on the inner surface of the tube then there is a bubble development zone as displayed in Figure 2Right, followed by the above transitions as one goes further up the tube, see Figure 3 Left. In all these situations there is a thin film of vapour separating the liquid from the tube surface. Of major importance in context is that the heat transfer rate per unit sectional area varies dramatically depending on the flow regime; a factor of 2 to 3 is typical, see Figure 3 Right. Although these results correspond to an air in water situation, similar flow regimes are likely in the massecuite case.

\section{Calandria tube flow models}

Two models are developed here: a crude 1D model whose basic aim was to identify the important dimensional groups in the bubbly flow region and above, and a 2D model which attempts to extract flow details in the lower region of the tubes.

\subsection{A crude 1D model of flow in the bubbly region and above}

The flow transitions described above have been observed in the Rouillard [4], Rein [6] and Echeverri [11] corn syrup tube experiments, and fluctuations in pressure over periods of about 2 secs with bubble explosions at the free surface were observed. As indicated earlier observations suggest a greatly reduced heat transfer rate under HTB conditions so this strongly suggests that the effect of impurities is to change the flow regime from a saturated 
flow boiling regime through to a subcooled flow regime or even liquid forced convection regime as depicted in Figure 3. Such changed behaviour could occur either because of viscosity changes in HTB or because of a reduced bubble production rate in HTB. We will set up models to identify relevant parameters. First a few observations and assumptions concerning the process will be presented.

The calandria is a clever, but also 'delicate', device that inputs heat throughout the liquid in the pan using the very large surface area of the many tubes that make up the device. Note the following connected processes:

- Bubbles will first be released from nucleation points on the surface of the tubes (the wall bubbles region). The actual bubble numbers (and size) released from the tube surface will depend on the roughness of the tubes and on-site experimental work would be required to estimate this. Further up the tubes (homogeneous) nucleation can occur within the tube (the core bubbles flow region). In either case the actual volume of water vapour released per unit time should be predictable using estimates (obtained experimentally) for the effective latent heat of the massecuite.

- The bubbles exert a body (buoyancy) force on the liquid and thus accelerate the movement of liquid through the tube. In fact this appears to be the only driving force for the convective flow in and out of the calandria. The large volumetric change due to state change will lift bubbles off the calandria tube surfaces towards the core of the tube.

- Under normal operating conditions heating causes boiling close to the interior faces of these many tubes so that the interior surfaces are bathed in water vapour which has a very small viscosity. This lubricates the surface, thus greatly facilitating the upward movement of liquid through the tube.

- Additionally this vapour layer thermally insulates the liquid from the high temperature $\left(100-110^{\circ} \mathrm{C}\right)$ on the surface of the tubes; such high surface temperatures would melt the sucrose (melting point $96^{\circ} \mathrm{C}$ ) and cause adhesion to, and possibly burning on, the surface.

- The released and moving bubbles also facilitate the movement of heat generated at the tube surface towards the interior of the tube so that state change will (normally) occur across the section of the tube; the conductivity of massecuite (or water vapour) is too small to distribute the heat to anything other than a small layer close to the tube walls.

- Under normal, relatively low viscosity conditions, bubbles move freely but under the abnormally high viscous conditions associated with HTB they cannot, so that the 


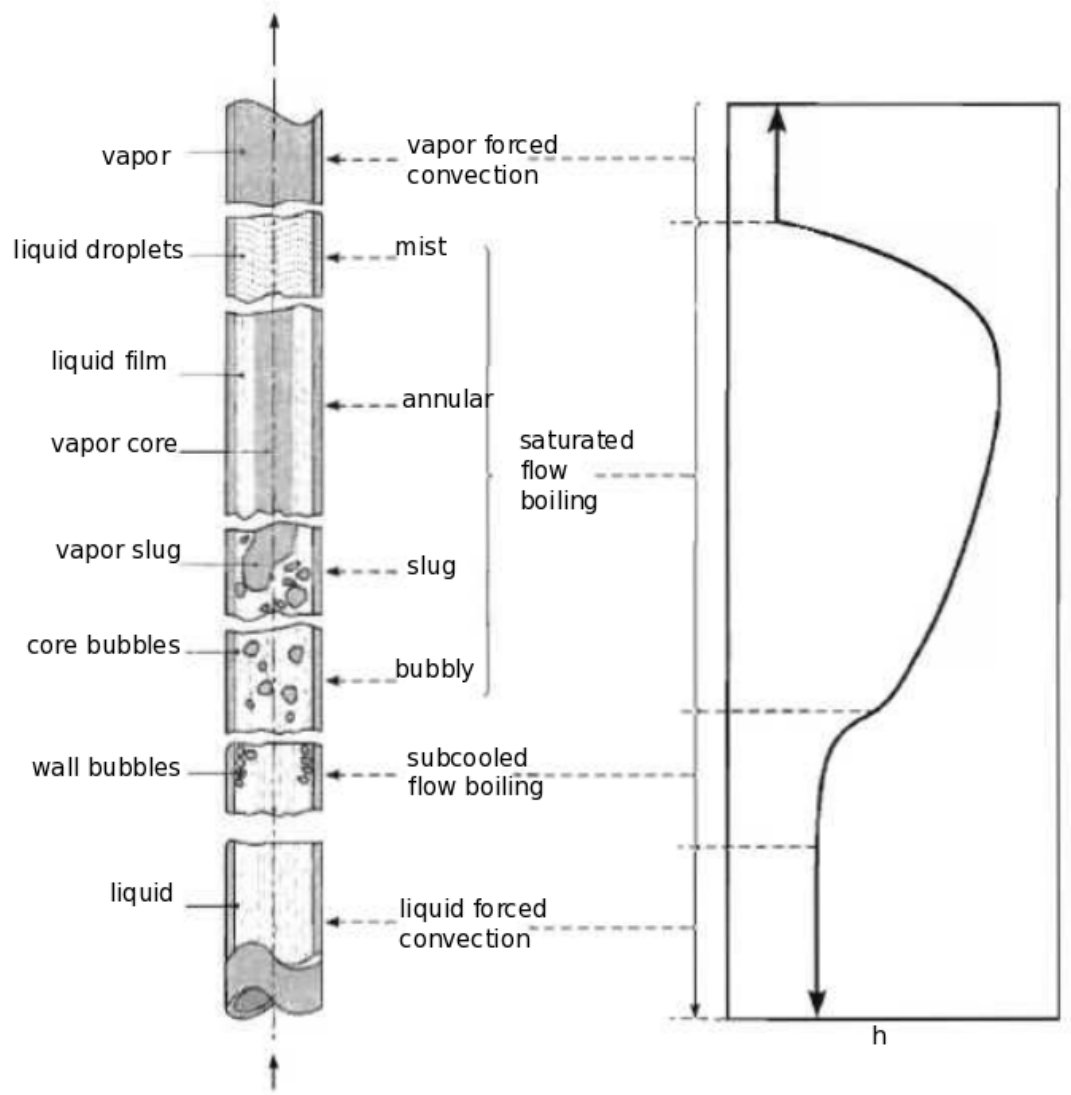

Figure 3: Various flow (left) and heat transfer (right) regimes associated with boiling in a tube. Note the large changes in heat transfer that occurs under 'saturated flow' conditions. 
heat transfer/state change processes may be confined to a thin layer close to the tube surface.

- Now if bubbles are not produced at the rate required to lubricate and insulate the walls then the lubrication layer is lost so the wall drag increases dramatically (a factor of $10^{3}$ ) and the movement of massecuite through the system slows down enormously. Bubbles also serve to redistribute heat to the massecuite thus reducing its viscosity. Furthermore, the external convective current normally feeding the tubes with massecuite 'will stop' because it is the tube flow that drives the system. Rather than having the tubes driving the system we now have a situation in which the external flow needs to push (or shove) massecuite through the tubes.

We can see from the above that if bubbles are not produced at the required rate several interdependent processes will collapse. Evidently the secret is to introduce agents that will facilitate the release of bubbles under HTB conditions, and this will be addressed in Sections 3. Here we address the question: "how can one improve the process under less than perfect (including stuck) conditions?" Specifically we require a model to determine the water vapour and massecuite flux out a calandria tube as a function of its length $L$, radius $a$, the heat exchange characteristics of the calandria tube, and massecuite thermal (importantly state change) and flow properties. It is assumed that the steam production unit is sufficiently powerful and efficient to keep the inside temperature of the tubes at $T_{m}$ $\left(110^{\circ} \mathrm{C}\right)$.

A complete analysis would require consideration of the mass, momentum and energy equations for the two phases, as well as the state equation for the water vapour, and due account would need to be taken of the momentum exchange between the two phases (given that velocities in the two phases will not generally be the same) and also the energy exchange. Such an analysis would require empirical input to account for this interaction between the phases which is not available for massecuite; suitably designed experiments, building on the work of Egglesston et al [12] are required. Here we will undertake a simpler crude analysis based on the above observations and other simplifying assumptions:

- We will assume the massecuite is at the temperature of boiling $T_{b}$ so that the additional heat flow from the tube walls just provides the latent heat needed to extract water vapour. Thus the temperature profile is assumed to be uniform across the tube except for a thin vapour film across which latent heat is supplied. The fraction of volume occupied by vapour will of course vary along the tube.

- We also assume a flat velocity profile separated from the tube boundary by the thin vapour film; under the turbulent two phase flow conditions expected this is likely to be the case on average. 
- We assume the 'bubbles' and the liquid massecuite move with the same velocity; in essence the bubbles are thought to 'carry' the liquid out of tube. This assumption greatly simplifies the calculations in that the liquid massecuite plus water vapour particles can be thought of as being consolidated single unit moving though the tube; we have effectively single phase flow with varying density along the tube.

- As a further simplification we assume that the viscous drag due to the this vapour film is negligible; this being the case we simply have buoyancy driven particle flow.

- A quasi-steady flow is assumed.

The above approximations are likely to be reasonable in the bubbly flow region of the tube and above. Below this region the a 2D flow model is appropriate, see Section 2.2.

\section{Momentum input due to bubbles}

The buoyancy uplift is due to the bubbles and this causes a change in momentum of the (liquid) massecuite and the water vapour, however, the momentum transport associated with the vapour is small, so that to first order we have

$$
\left[\left(\rho_{l} \pi a^{2} V_{l}(1-\alpha)\right) V_{l}\right]_{x} \approx\left(\alpha \pi a^{2}\right)\left(\rho_{l}-\rho_{v}\right) g, \approx\left(\alpha \pi a^{2}\right) \rho_{l} g
$$

which reduces to

$$
\left[(1-\alpha) V_{l}^{2}\right]_{x}=\alpha g
$$

where $\alpha(x)$ is the volume fraction of vapour, and $V_{l}(x)$ is the massecuite velocity up the tube at location $x$ from the bottom of the tube, and $\left(\rho_{l}, \rho_{v}\right)$ are the massecuite and vapour densities respectively. Note that the change in density of that portion of the massecuite that is converted to vapour is $\left(\rho_{l}-\rho_{g}\right) \approx \rho_{l}$ so that the uplift per unit volume acting on the massecuite is approximately $\rho_{l} g \alpha$.

\section{State change}

The heat necessary to drive the state change is provided by the tube walls at temperature $T_{w}$ (assumed constant) so that

$$
(2 \pi a) h\left(T_{w}-T_{b}\right)=-\left[\pi a^{2}(1-\alpha)\right]_{x} V_{l}\left(\rho_{l}-\rho_{v}\right) \mathcal{L},
$$

which gives

$$
2 h\left(T_{w}-T_{b}\right)=-a[(1-\alpha)]_{x} V_{l} \rho_{l} \mathcal{L},
$$


where $\mathcal{L}$ is the 'effective latent heat' ${ }^{2}$ per unit mass of the massecuite. Note that the heat exchange coefficient $h\left(V_{l}\right)$ will also change as the flow regime changes from quiet bubbling through to slug flow.

We have two equations in $\alpha(x), V_{l}(x)$. The appropriate scales to introduce are

$$
x=L x^{\prime}, V_{l}=V_{0} V_{j}^{\prime}, h=h_{0} h^{\prime}\left(V_{l}^{\prime}\right),
$$

where

$$
V_{0}=\sqrt{\alpha_{0} g L}
$$

is the expected buoyancy induced velocity scale based on a typical scale for $\alpha$. The equations reduce to (dropping primes):

$$
\begin{gathered}
{\left[(1-\alpha) V^{2}\right]_{x}=1,} \\
\alpha_{x}=h \xi
\end{gathered}
$$

where

$$
\xi=\frac{2 h_{0}\left[T_{w}-T_{b}\right]}{a V_{0} \rho_{l} \mathcal{L}}
$$

is the important dimensionless group of the problem. The appropriate boundary conditions to impose are

$$
V(0)=V_{0}, \quad \alpha(0)=0 .
$$

Comment: The above system is incomplete. Matching onto the external flow would be required to determine $V_{0}$, and also $(\alpha, \mathcal{L})$ levels would need to be updated as the massecuite is cycled through the calandria, however we believe that the important physics is contained in the above equations. The thought is that the primary mechanisms of state change and buoyancy uplift are effectively quantified in the above equations, so that it is the "thermodynamic performance' parameter $\xi$ that quantifies the efficiency of the calandria boiling process as a function of the relevant parameters. The above equations can be integrated for suitable heat transfer functions $h(V)$ however more insight can be gained by simply assuming $h$ remains constant (and very different) in the various flow regimes as seen in Figure 3.

Evidently, see (5), in any of the constant $h$ flow regimes, there is a linear increase in the amount of released vapour along the tube (proportionality factor $\xi$ ), with a consequent linear increase in liquid momentum as seen in $(4)$. Note that $\xi$ increases in proportion to $h_{0} / \mathcal{L}$, so that, either if the latent heat required to convert the massecuite increases or the heat transfer through the walls of the calandria tube reduces, there will be reduction in boiling rate. Of course the latter occurs dramatically when there is a change in flow regime as displayed in Figure 3, and one would expect both effects to occur simultaneously for reasons described earlier; it is no surprise that 'getting stuck' is a dramatic effect.

\footnotetext{
${ }^{2}$ really the heat required to release the water
} 
There is a factor of about three in the heat exchange rate in the tube as the flow changes from subcooled boiling through to slug flow and then saturated flow boiling, see Figure 3, so that even in the absence of latent heat changes there will be a dramatic change in the boiling process. The Eggleston et al experiments [12] suggest that slug flow happens normally but that under HTB conditions subcooled boiling is likely; HTB massecuite behaviour might be expected. Useful experimental results are needed to determine the value of the thermal efficiency parameter $\xi$ for each of the fluid flow regimes indicated in Figure 3. It should be noted that the above model did not take into account the viscous drag acting on massecuite moving through the tubes, the rationale being that the drag will be small provided the lubricating film is present. If the film is not present then of course the viscous drag will be large, but more importantly the massecuite will make contact with the hot tube walls and will burn, so the process will certainly get stuck.

\subsection{A two-dimensional model of the flow into the calandria}

As displayed in Figure 2 Right and Figure 3, flow in the entry region of a calandria tube will involve heat transfer by forced liquid convection and will be two-dimensional, with a boundary layer growing away from the flow entry point at $x=0$. Separating the massecuite from the tube will be a thin vapour film of thickness $h(x)$ across which heat will be transferred from the walls, see Figure 4. This heat transfer will cause the liquid massecuite to boil, releasing water vapour. Above this region will be a wall bubble region followed by a core bubble region and then other regions as described earlier. These flows will be coupled to a convective flow external to the calandria; it is a complicated problem.

However, in the lower region of interest here the flow regions are thin, so that lubrication type approximations are possible and useful analytic results are available. Thus, assuming steady state, in the liquid region, $h<y<H$, we have

$$
\frac{-1}{\rho_{l}} \frac{\partial P_{l}}{\partial x}+\nu_{l} \frac{\partial^{2} u_{l}}{\partial y^{2}}+g=0
$$

with

$$
\frac{\partial P_{l}}{\partial y}=0
$$

which implies that

$$
P_{l}=P_{l}(x),
$$

where $u_{l}(x, y)$ is the velocity along the tube and $P_{l}(x)$ is the pressure in the liquid. This integrates to give

$$
u_{l}(x, y)=\frac{1}{2}\left[\frac{1}{\rho} \frac{\partial P_{l}}{\partial x}-g\right] y^{2}+A(x) y+B(x)
$$




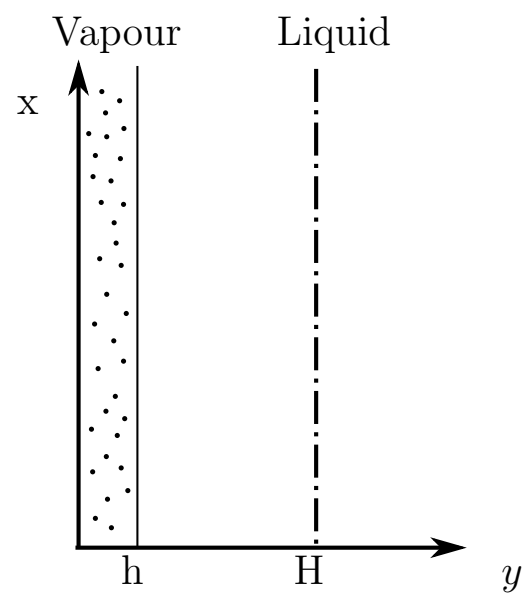

Figure 4: The physical model near the lower tube.

In the vapour region, $y<h$, we have

$$
\frac{-1}{p_{v}} \frac{\partial P_{v}}{\partial x}+\nu \frac{\partial^{2} u_{v}}{\partial y^{2}}=0
$$

where $u_{v}(x, y)$ is the velocity down the tube and $P_{v}(x)$ is the vapour pressure with

$$
\frac{\partial P_{v}}{\partial y}=0
$$

which implies that

$$
P_{v}=P_{v}(x)
$$

Equation (10) integrates to give

$$
u_{v}=\frac{1}{2 \nu P_{v}}\left(\frac{\partial P_{v}}{\partial x}\right) y^{2}+C(x) y+D(x) .
$$

Water vapour is created (and liquid lost) at the interface $h(x)$ of the two regions at a rate that is dependent on the heat transfer rate from the tube walls and the Latent heat $L_{v}$ associated with the state change. These mass conservation and heat flux and Stefan conditions require

$$
\rho_{v}\left[v_{v}-u_{v} \frac{\partial h}{\partial x}\right]=\rho_{l}\left[v_{l}-u_{l} \frac{\partial h}{\partial x}\right]=-k_{v} \frac{T_{w}-T_{b}}{h L_{v}} .
$$

Additionally we require that $u_{v}(0, y)=0$ and $u_{l}(H, y)=0$, and a pressure continuity condition (or equivalent) will be required at the vapour/liquid interface $h(x)$. The unknown 
coefficients $A(x), B(x), C(x), D(x)$ as well as the vapour film thickness $h(x)$ need to be determined so that all these conditions are satisfied and additionally some consideration of the temperature development along the tube may be necessary. It seems likely that the effect of containment in the tube will be to constrain the vapour film thickness to a constant value which will be determined by a common liquid and vapour pressure across the tube, but additional analysis will be required to clarify this issue. The analysis is ongoing.

\section{Possible explanations for hard to boil massecuite}

\subsection{Introduction}

To identify the key factors which prevent the boiling of massecuite, [12] have carried out a comprehensive set of experiments that examine the physical characteristics of normal and hard-to-boil (HTB) massecuite. These experiments show, in particular, that HTB massecuite has (i) significantly reduced thermal conductivities (by up to 30\%) and heat transfer coefficients, and (ii) can exhibit viscoelastic rheology (Figure 5) due to the presence of an intermolecular gel-like network that is formed by long-chained polysaccharides. The role of the polysaccharide network is two-fold. Firstly, it can influence the energetic requirements for the nucleation of a vapour bubble. Secondly, vapour bubbles can be entrapped within the polysaccharide matrix, thus making it appear as if the massecuite is not boiling. In this

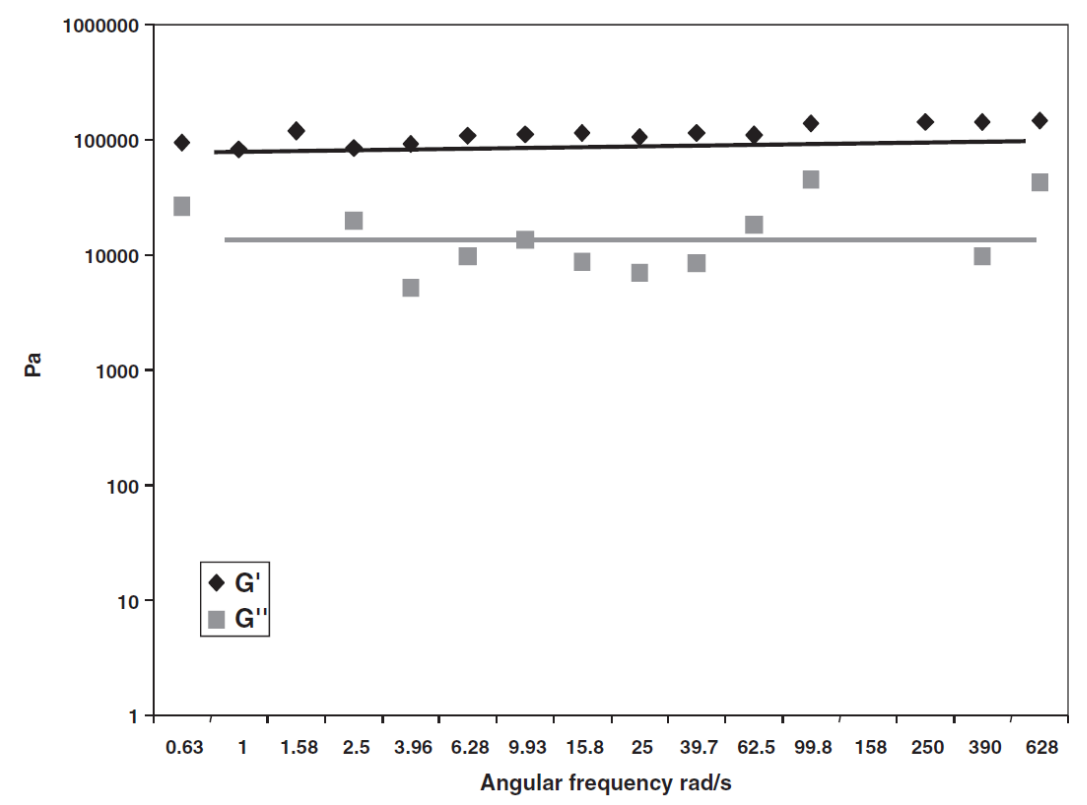

Figure 5: Storage $\left(G^{\prime}\right.$, black diamonds) and loss $\left(G^{\prime \prime}\right.$, grey squares) modulii for HTB massecuite showing substantial elastic behaviour $\left(G^{\prime \prime} \ll G^{\prime}\right)$. Figure taken from [12]. 
section of the report, simple models are developed in order to explore how the boiling time is affected by reduced thermal properties and viscoelasticity affects on the onset of a vapour bubble.

\subsection{Thermal modelling}

The industrial boiler (Figure 1) is a cylindrical system which heats massecuite radially from a lateral steam advection. It has been reported in literature ([11] and [12]) that the composition of the massecuite can significantly alter its thermal conductivity as well as the advective heat transfer coefficient with the steam. Since the boiling process relies on the energy transfer from steam to massecuite, the boiling time can potentially be significantly altered by changes in the thermal properties. It is therefore of interest to model the thermal transfer and investigate the time to boil for a set of conductivity and heat transfer parameters.

\section{Model development}

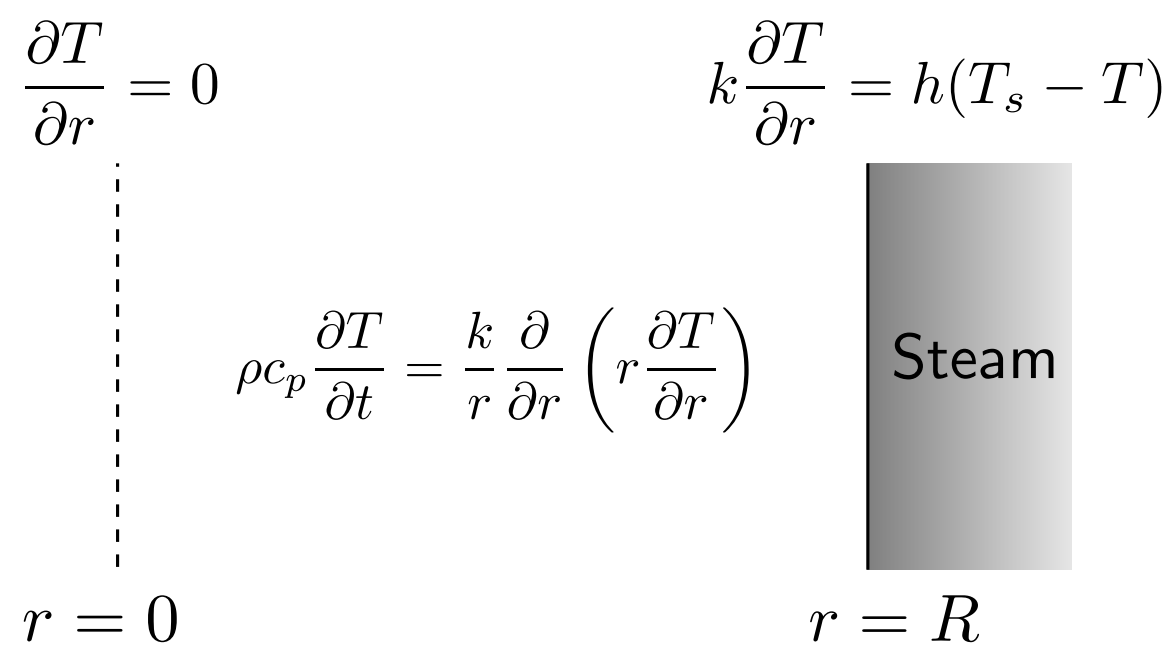

Figure 6: Geometric setup of the thermal problem to be solved.

We consider a cylindrical tube of radius $R$ centred at the origin full of massecuite with steam contact at $r=R$. We will solve the heat equation in the massecuite under the assumption that there are no azimuthal or vertical temperature variations ${ }^{3}$. The temperature variation within the massecuite is governed by

\footnotetext{
${ }^{3}$ Realistically, the temperature may vary vertically due to the steam cooling and condensing but we will assume the system is designed so that sufficient energy is available for transfer along the entire length of the pipe.
} 


$$
\rho c_{p} \frac{\partial T}{\partial t}=\frac{k}{r} \frac{\partial}{\partial r}\left(r \frac{\partial T}{\partial r}\right)
$$

where $\rho$ is the massecuite density, $c_{p}$ is the thermal heat capacity at constant pressure, and $k$ is the thermal conductivity of massecuite. For boundary conditions we will take a symmetry condition $T_{r}=0$ at $r=0$ and apply a Newton's law of cooling with the steam contact,

$$
k \frac{\partial T}{\partial r}=h\left(T_{s}-T\right), \quad r=R
$$

where $h$ is the advective heat transfer coefficient with the steam, and $T_{s}$ is the steam temperature. The setup is shown in Figure 6.

We will take as an initial condition $T(r, 0)=T_{a}$ where $T_{a}$ is the ambient temperature of the massecuite as it enters the boiler. Table 1 lists typical values for the model parameters. We are interested in solving the model and computing the time to boil which we will define as the time when the midpoint $R / 2$ reaches the vaporisation temperature $T_{v}$. We nondimensionalize (13) using the scales

$$
r \sim R, \quad t \sim \frac{R^{2} \rho c_{p}}{k}, \quad T=T_{s}-\left(T_{s}-T_{a}\right) u
$$

which leads to the reduced model

$$
\begin{gathered}
\frac{\partial u}{\partial t}=\frac{1}{r} \frac{\partial}{\partial r}\left(r \frac{\partial u}{\partial r}\right), \\
\left.\frac{\partial u}{\partial r}\right|_{r=0}=0,\left.\quad \frac{\partial u}{\partial r}\right|_{r=1}=-\alpha u ; \quad u(r, 0)=1,
\end{gathered}
$$

with parameter $\alpha=h R / k$ representing the effective advective heat transfer.

\section{Boiling time calculation}

We solve (14) using separation of variables which leads to

$$
u(r, t)=\sum_{n=1}^{\infty} A_{n} \exp \left(-\lambda_{n} t^{2}\right) J_{0}\left(\lambda_{n} r\right), \quad A_{n}=\frac{\int_{0}^{1} r J_{0}\left(\lambda_{n} r\right) \mathrm{d} r}{\int_{0}^{1} r J_{0}\left(\lambda_{n} r\right)^{2} \mathrm{~d} r}
$$




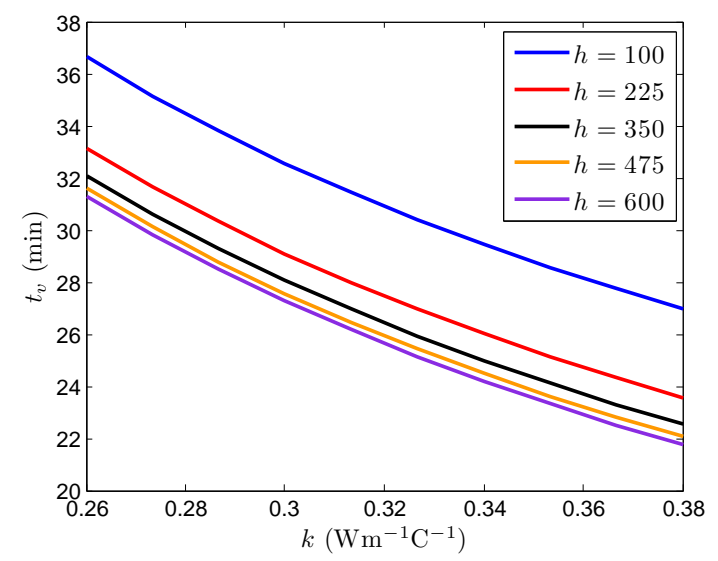

(a) One term expansion.
Hard to boil massecuite

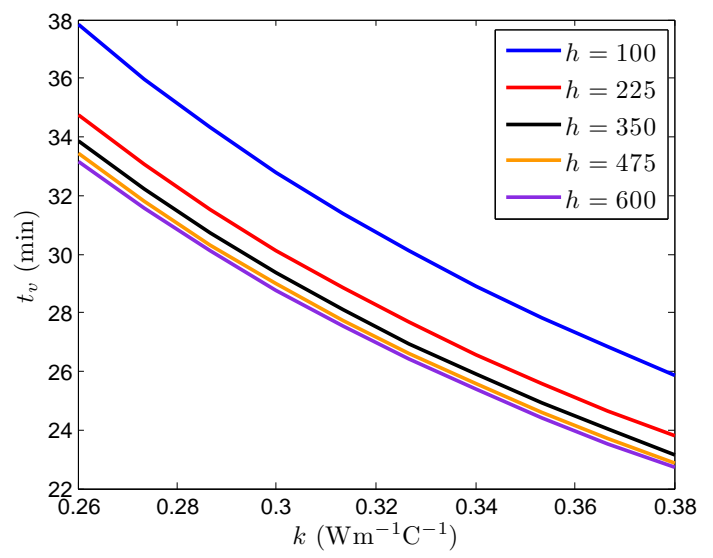

(b) Ten term expansion.

Figure 7: Vaporisation times for a selection of advective heat transfer coefficients, $h$, and thermal conductivities $k$. The one term expansion comes from using (18) while the ten term expression comes from numerically solving (15) with ten terms.

where $J_{n}(x)$ is the $n^{\text {th }}$ Bessel function of the first kind. Each eigenvalue, $\lambda_{n}$, satisfies the transcendental equation,

$$
\lambda_{n} J_{1}\left(\lambda_{n}\right)=\alpha J_{0}\left(\lambda_{n}\right) .
$$

The boiling or vapourisation time $t_{v}$ occurs when $u\left(\frac{1}{2}, t_{v}\right)=u_{v}$ where,

$$
u_{v}=\frac{T_{s}-T_{v}}{T_{s}-T_{a}}=0.7273
$$

and if we consider a one term expansion of the Fourier series (15) then we can derive an analytical expression for $t_{v}$,

$$
t_{v}=\frac{1}{\lambda_{1}^{2}} \log \left(\frac{J_{0}\left(\frac{\lambda_{1}}{2}\right) A_{1}}{u_{v}}\right)
$$


Table 1: Parameter values used to calculate the boiling time of a cylindrical volume of massecuite that is being heated from the side.

\begin{tabular}{|c|c|c|c|}
\hline Parameter & Description & Value & Reference \\
\hline$R$ & Calandria tube radius & & Provided \\
\hline$\rho$ & Massecuite density & $1400 \mathrm{~kg} \mathrm{~m}^{-3}$ & $[1]]$ \\
\hline$c_{p}$ & Thermal heat capacity & $2000 \mathrm{~J} \mathrm{~kg}^{-1} \mathrm{C}^{-1}$ & {$[10]$} \\
\hline$k$ & Thermal conductivity & $0.26-0.38 \mathrm{~W} \mathrm{~m}^{-1} \mathrm{C}^{-1}$ & {$[12]$} \\
\hline$h$ & Advective heat transfer coefficient & $100-600 \mathrm{~W} \mathrm{~m}^{-2} \mathrm{C}^{-1}$ & {$[11]$} \\
\hline$T_{s}$ & Steam temperature & $110 \mathrm{C}$ & {$[11]$} \\
\hline$T_{a}$ & Ambient massecuite temperature & $55 \mathrm{C}$ & {$[11]$} \\
\hline$T_{v}$ & Vaporisation temperature & $70 \mathrm{C}$ & $[1]]$ \\
\hline
\end{tabular}

We plot vaporisation times for a range of $h$ and $k$ as given in Table 1 in Figure 7a for the single term expression and Figure $7 \mathrm{~b}$ for a numerical solution of $u\left(\frac{1}{2}, t_{v}\right)$ with ten terms using (15). The two are in fairly good agreement with one another. As expected, there is a decrease in the boiling time with an increase in the heat transfer coefficient. The relationship between boiling time and thermal conductivity can be explained by first recalling that the boiling time is calculated at a point within the massecuite. Therefore, larger thermal conductivities will lead to a faster transfer of heat from the wall to that point, thereby reducing the time that is required for the temperature to reach the boiling point.

The results obtained indicate a vaporisation time of the order of 20 to 40 minutes which is consistent with the typical processing times for massecuite. It can be seen from Figure $7 \mathrm{~b}$ that the effect of a reduction of massecuite conductivity of the order typically occurring under HTB conditions is to change to processing time from 28 mins to about 38 mins. This is significant but not as large as observed in practice; this is unlikely to be the explanation for the problem.

Note, see $(17,18)$, that an increase change in the vaporisation temperature $T_{v}$ will cause a logarithmic increase in the vaporisation time $t_{v}$; such a change is likely for HTB massecuite.

\subsection{Bubble nucleation in a viscoelastic medium}

Classical (homogeneous) nucleation theory describes the energetic requirements that must be satisfied in order to form a vapour bubble from a liquid. In essence, the radius of the nucleated bubble must be sufficiently large that the energy that is released from the phase transformation can offset the energy that is required to form a liquid-vapour interface. In the case of a bubble nucleating in a viscoelastic medium, there is an additional energy barrier that must be overcome; this is the elastic energy that is required to compress the viscoelastic medium due to the volumetric expansion of liquid as it transforms into gas. In this section, we will explore how the elastic energy affects the critical radius of nucleation. 


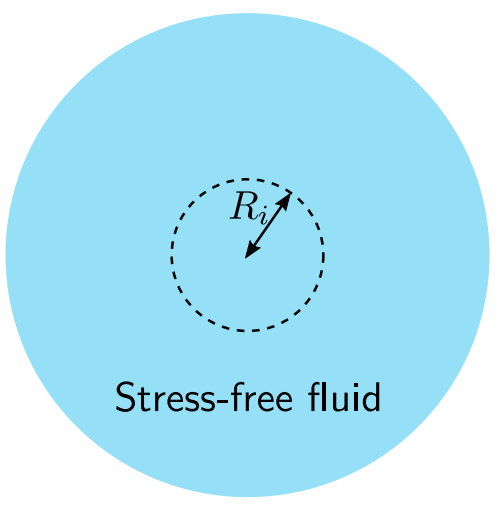

(a)

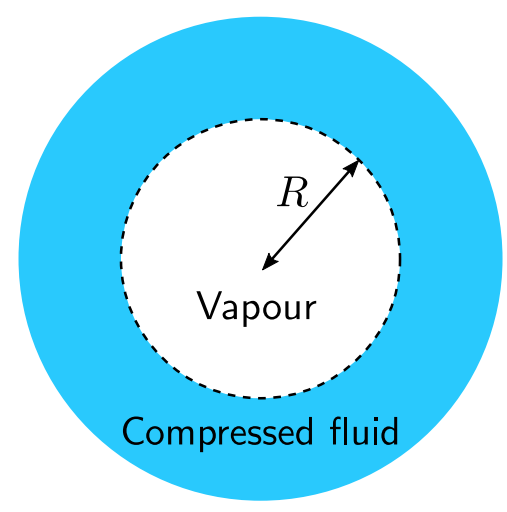

(b)

Figure 8: A schematic diagram of bubble nucleation in a viscoelastic medium. When a spherical volume of liquid with radius $R_{i}$ undergoes vaporisation (a), it expands to a sphere of radius $R=\left(\rho_{f} / \rho_{v}\right)^{1 / 3} R_{i}$ due to the density of fluid, $\rho_{f}$, being greater than the density of vapour $\rho_{v}$. This expansion induces a compressive strain in the surrounding viscoelastic fluid. The elastic energy associated with this deformation can affect the critical radius of nucleation.

We consider the nucleation of a spherical bubble in an infinite bath of viscoelastic fluid (massecuite), as shown in Figure 8. The system is assumed to be axisymmetric; therefore, the variables of the model only depend on the radial co-ordinate $r$, where $r=0$ corresponds to the centre of the vapour bubble. Furthermore, we assume that the volume of fluid that undergoes vaporisation is a sphere of radius $R_{i}$. Since mass is conserved during the phase transformation, the radius of the vapour bubble can be calculated as

$$
R=\left(\rho_{f} / \rho_{v}\right)^{1 / 3} R_{i}
$$

where $\rho_{f}$ and $\rho_{v}$ denote the densities of the fluid and vapour, respectively. Thus, material points surrounding the vaporising liquid are displaced by an amount

$$
u_{b}=R-R_{i}=\left[1-\left(\rho_{v} / \rho_{f}\right)^{1 / 3}\right] R .
$$

Despite the fact that large strains may occur due to the volumetric expansion, the mechanics of the system are described using linear elasticity; that is, geometric nonlinearity is not considered. This is solely for simplicity; extending the model is in principle straightforward, see later.

The energy change that occurs upon nucleating a spherical bubble of radius $R$ can be written as 


$$
\Delta E=-U_{\text {vap }}+U_{\text {surf }}+U_{\mathrm{e}}
$$

where $U_{\text {vap }}, U_{\text {surf }}$, and $U_{\mathrm{e}}$ correspond to the vaporisation, surface, and elastic energies, respectively. The energy of vaporisation is

$$
U_{\text {vap }}=\frac{4}{3} \pi R_{i}^{3} \rho_{f} L_{v}
$$

where $L_{v}$ is the latent heat of vaporisation. In terms of the radius of the bubble this becomes

$$
U_{\text {vap }}=\frac{4}{3} \pi R^{3} \rho_{v} L_{v}
$$

The surface energy is given by

$$
U_{\text {surf }}=4 \pi R^{2} \gamma_{l v}
$$

where $\gamma_{l v}$ is the surface tension of the fluid-vapour interface. In the framework of linear elasticity, the elastic energy of a uniaxial, axisymmetric deformation of the form

$$
\boldsymbol{u}(r, \theta, \phi)=u(r) \boldsymbol{e}_{r}
$$

can be written using the summation convention as

$$
U_{\mathrm{e}}=4 \pi \int_{R}^{\infty}\left[\frac{1}{2} \lambda\left(\mathrm{e}_{k k}\right)^{2}+\mu \mathrm{e}_{i j} \mathrm{e}_{i j}\right] r^{2} \mathrm{~d} r,
$$

where $\lambda$ is the Lame constant, $\mu$ is the shear modulus, and the principal strains are given by

$$
\mathrm{e}_{r r}=\mathrm{d} u / \mathrm{d} r, \quad \mathrm{e}_{\theta \theta}=\mathrm{e}_{\phi \phi}=u / r .
$$

Calculating the elastic energy (23) requires solving the equations of linear elasticity for the displacement $u$. Conservation of linear momentum in axisymmetric spherical geometry leads to

$$
(\lambda+2 \mu) \frac{\mathrm{d}}{\mathrm{d} r}\left[\frac{1}{r^{2}} \frac{\mathrm{d}}{\mathrm{d} r}\left(r^{2} u\right)\right]=0 .
$$

The displacement at $r=R$ is fixed by conversation of mass arguments (see previous discussion). Furthermore, we assume that the displacement decays to zero in the far field. Therefore, appropriate boundary conditions for this problem are

$$
\begin{array}{ll}
u=\left[1-\left(\rho_{v} / \rho_{f}\right)^{1 / 3}\right] R, & r=R, \\
u \rightarrow 0, & r \rightarrow \infty .
\end{array}
$$


The solution to (24) and (25) is given by

$$
u(r)=\left[1-\left(\frac{\rho_{v}}{\rho_{f}}\right)^{1 / 3}\right] \frac{R^{3}}{r^{2}},
$$

and the elastic energy that is associated with this deformation is

$$
U_{\mathrm{e}}=8 \pi \mu\left[1-\left(\frac{\rho_{v}}{\rho_{f}}\right)^{1 / 3}\right]^{2} R^{3} .
$$

Assembling the various energy components we see from (19) that

$$
\frac{3}{4 \pi} \Delta E=-R^{3}\left[\rho_{v} L_{v}-6 \mu\left[1-\left(\rho_{v} / \rho_{f}\right)^{1 / 3}\right]^{2}\right]+3 R^{2} \gamma_{l v}
$$

The critical bubble radius, $R_{c}$, corresponds to the radius at which it becomes energetically favourable (i.e., $\Delta E$ decreases) for a bubble to grow rather than shrink. Therefore, $R_{c}$ can be calculated by maximising the change in energy $\Delta E$ with respect to $R$. There will exist such a turning point, and thus a critical radius, only if the coefficient of the $R^{3}$ is negative which requires

$$
6 \mu\left[1-\left(\rho_{v} / \rho_{f}\right)^{1 / 3}\right]^{2}<\rho_{v} L_{v},
$$

that is, the shear modulus $\mu$ needs to be small enough.

For sufficiently small elastic modulus $\mu$ there exists a critical radius given by

$$
R_{c}=\frac{2 \gamma_{l v}}{\rho_{v} L_{v}-6 \mu\left[1-\left(\rho_{v} / \rho_{f}\right)^{1 / 3}\right]^{2}}
$$

which reduces to

$$
R_{0}=2 \gamma_{l v} /\left(\rho_{v} L_{v}\right)
$$

in the absence of an elastic resistance to bubble formation. The effect of an increase of elastic resistance is to increase the critical radius required for bubble growth, with this result becoming infinite when the elastic resistance exceeds $\mu_{\text {crit }}$ given by

$$
\frac{6 \mu_{c r i t}\left[1-\left(\rho_{v} / \rho_{f}\right)^{1 / 3}\right]^{2}}{\rho_{v} L_{v}}=1,
$$

The implication is that the probability of bubble formation is reduced for increasing $\mu$ until the critical value $\mu_{\text {crit }}$ is reached; after that bubble growth is impossible.

Using the values $\rho_{v}=0.2 \mathrm{~kg} / \mathrm{m}^{3}, \rho_{f}=10^{3} \mathrm{~kg} / \mathrm{m}^{3}, L_{v}=10^{6} \mathrm{~J} / \mathrm{kg}$, with a value of $\mu=10^{5} \mathrm{~Pa}$, we find

$$
\left[1-\left(\rho_{v} / \rho_{f}\right)^{1 / 3}\right]^{2}=0.88
$$


so that the critical shear viscosity is given by

$$
\mu_{\text {crit }} \approx \rho_{v} L_{v} / 6=0.37 \cdot 10^{5} \mathrm{~Pa}
$$

Now typical values of $\mu$ for HTB massecuite are of the order of $10^{5} \mathrm{~Pa}$, so that the above theory would indicate that the critical shear modulus values are exceeded, so that bubbles will not form!

The above theory was based on a linear elastic response, however on dimensional grounds one can claim that the elastic energy contribution to $\Delta E$ under non-linear elastic circumstances will be of the form

$$
U_{e}=\mu R^{3} \cdot \operatorname{fn}\left(\rho_{v} / \rho_{f}, R / R_{n l}\right)
$$

where $\mu$ corresponds to the small strain limit and the function $\mathrm{fn}()$ accounts for non-linear elastic effects which become important when $R \approx R_{n l}$. The dependence on $\rho_{v} / \rho_{f}$ can be ignored. Note especially the $R^{3}$ behaviour which is required by dimensionality. The observation is that elastic effects and vaporisation effects compete to determine the sign of the $R^{3}$ term in $\Delta E$, see (28), which we have seen determines whether or not bubbles will occur. If HTB massecuite is a shear stiffening material then non-linear effects will reduce $\mu_{c r i t}$, whereas in the shear weakening case the effect may enable bubbles to form.

In summary: yes rheological effects due to polysaccharide almost assuredly will suppress boiling.

\section{Conclusions}

A crude analysis of the flow up a calandria tube identified

$$
2 h\left(T_{w}-T_{b}\right) / a V_{0} \rho_{l} \mathcal{L}
$$

as the parameter determining the efficiency of the boiling process, so that changes in the effective latent heat of the massecuite or (more dramatically) changes in the heat transfer rate $h$ will strongly effect the calandria operation. The latter occurs when there is a flow regime change from slug or bubble flow (under normal conditions) through to subcritical flow boiling under HTB conditions.

The results from modelling flow of heat from the boiler into the bulk indicate that the boiling time is not significantly affected by reductions in the thermal conductivity and heat transfer coefficient of HTB massecuite. However, the results do indicate that changes in the boiling temperature can significantly affect the boiling time and, although not explicitly investigated, one would expect changes in the latent heat of vaporisation to strongly affect the onset of boiling; careful experiments are needed to see if these quantities vary from normal to HTB massecuite. In addition, the onset of fouling at the boiler wall could substantially 
affect the transfer of heat into the massecuite and hence alter the boiling time. Further investigations into the role of fouling could prove to be beneficial.

Our results do suggest that viscoelastic effects associated with polysaccharide contamination can prevent bubbles from forming and in fact the available data indicates that bubbles will not be formed in HTB massecuite.

Other effects may play a role but the strong indications are that the primary cause for HTP is polysaccharide contamination and the mechanism is bubble suppression.

\section{Acknowledgements}

Iain Moyles acknowledges the travel support from a Science Foundation Ireland grant SFI/13/IA/1923 to attend this workshop.

\section{References}

[1] Ziegler, J. Sugar boiling: some useful strategies. The Sugar Journal, 1979, catedras.facet.unt.edu.ar

[2] Koster, K. C., Vermeulen, P. L. M., Getaz, M. A., and Lionnet, G. R. E. Some notes on abnormal processing difficulties during spring. Proceedings South African Sugar Technologies Association. 52, 1978, 127-130.

[3] Saska, M. Heat transfer rates in boiling of cane syrups and molasses and the phenomenon of hard-to-boil massecuites. Proceedings Sugar Industry Technologists Meeting, Australia. 62, 2003, 175-187.

[4] Rouillard, E.E.A. Massecuite boiling. Proceedings South African Sugar Technologists Association., 59, 1985, 43-48.

[5] Eggleston, G., Côte, G., and Santee, C. New insights on the hard-to-boil massecuite phenomenon in raw sugar. Food Chemistry, 126, 2011, 21-30.

[6] Rein, P. W. Instability in pan boiling. ISSCT Processing Workshop, August, 2014, pp $1-19$.

[7] Butterworth, D. and Hewitt, G. F. Twp-phase Flow and Heat Transfer. Harwell series. Oxford University Press, 1977.

[8] Wallis, G. B. One-dimensional two-phase flow. McGraw-Hill, New York, 1970.

[9] Duffault, E. and Godshall, M. A. Molecular probes for assessing boiling difficulties. Sugar Processing Reaserch Institute, Conference on Sugar Processing, 2004, 403-416. 
[10] Asadi, M. Beet-sugar handbook. John Wiley \& Sons, 2006.

[11] Echeverri, L. F. Measurements and computations of the flow in full-scale sugarcrystallizers and in lab-scale models. PhD thesis, Louisiana State University, 2007.

[12] Eggleston, G., Côté, G, and Santee, C. New insights on the hard-to-boil massecuite phenomenon in raw sugar manufacture. Food Chemistry, 126, 2011, 21-30. 